\title{
Compensation of the Beam-Beam Effect in Proton-Proton Colliders
}

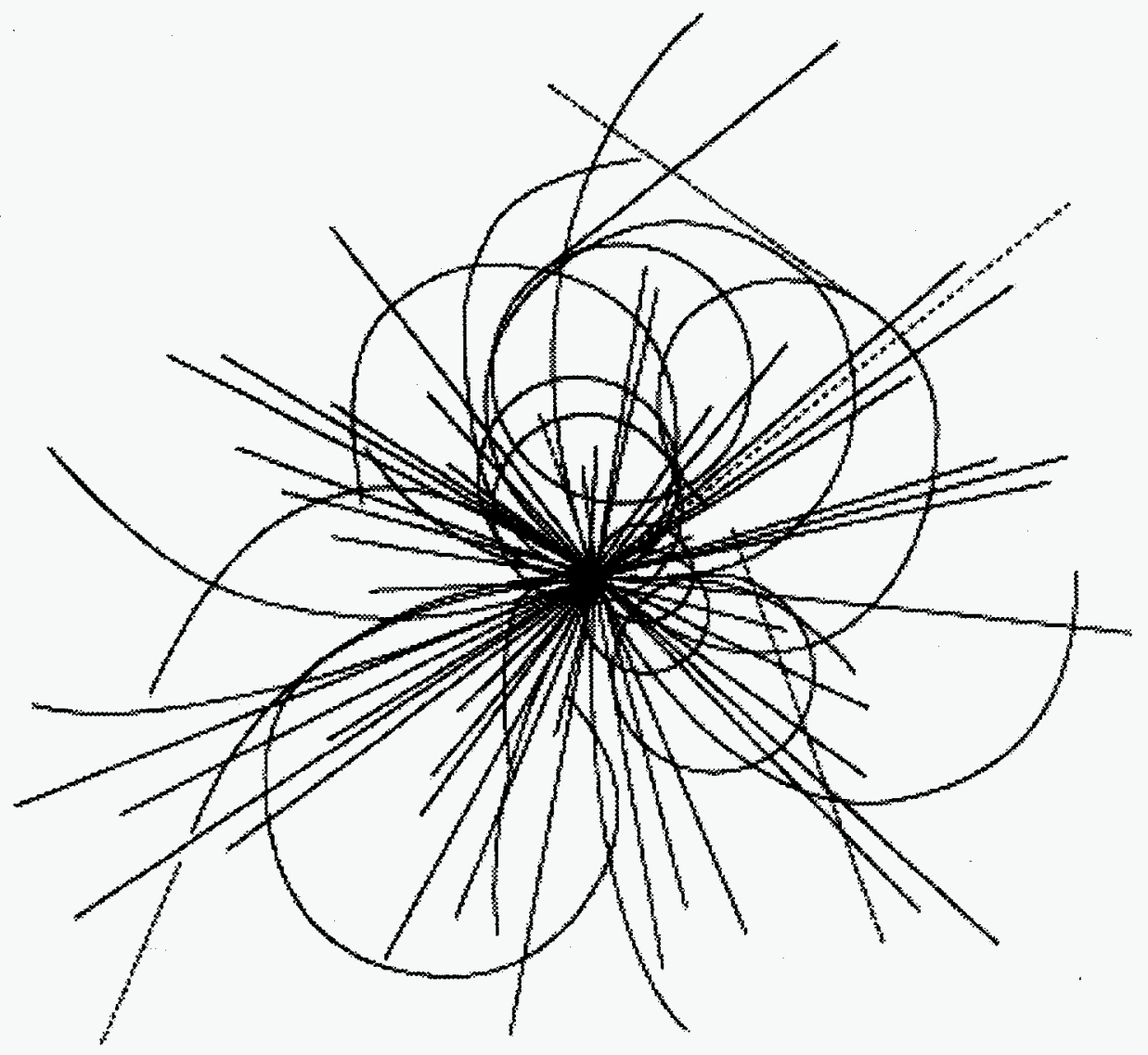

\section{Superconducting Super Collider Laboratory}
E. Tsyganov
R. Meinke
W. Nexsen

A. Zinchenko 


\section{Disclaimer Notice}

This report was prepared as an account of work sponsored by an agency of the United States Government. Neither the United States Government or any agency thereot, nor any of their employees, makes any warranty, express or implied, or assumes any legal liability or responsibility for the accuracy, completeness, or usefulness of any information, apparatus. product, or process disclosed, or represents that its use would not infringe privately owned rights. Reference herein to any specific commercial product, process, or service by trade name, trademark, manufacturer, or otherwise, does not necessarily constitute or imply its endorsement, recommendation, or favoring by the United States Govemment or any agency thereot. The views and opinions of authors expressed herein do not necessarily state or reflect those of the United States Govemment or any agency thereot. 


\section{DISCLAIMER}

Portions of this document may be illegible in electronic image products. Images are produced from the best available original document. 



\title{
Compensation of the Beam-Beam Effect in Proton-Proton Colliders*
}

\author{
E. Tsyganov, R. Meinke, and W. Nexsen \\ Superconducting Super Collider Laboratory ${ }^{\dagger}$ \\ 2550 Beckleymeade Ave. \\ Dallas, TX 75237
}

\author{
A. Zinchenko \\ Joint Institute for Nuclear Research \\ Dubna, Russia
}

October 1993

"To be submitted to Nuclear Instruments \& Methods in Physics Research.

Operated by the Universities Research Association, Inc., for the U.S. Department of Energy under Contract No. DE-AC35-89ER40486. 


\title{
COMPENSATION OF THE BEAM-BEAM EFFECT IN PROTON-PROTON COLLIDERS
}

\author{
E. Tsyganov, R. Meinke, and W. Nexsen \\ Superconducting Super Collider Laboratory* \\ 2550 Beckleymeade Ave. \\ Dallas, TX 75237 \\ A. Zinchenko \\ Joint Institute for Nuclear Research \\ Dubna, Russia
}

\begin{abstract}
Compensation of the beam-beam effect in high-energy proton-proton colliders using a low-energy electron beam is proposed. It is concluded that such compensation looks feasible. Requirements for such a device are formulated.
\end{abstract}

*Operated by the Universities Research Association, Inc., for the U.S. Department of Energy under Contract No. DE-AC35-89ER40486. 


\section{Introduction}

The head-on beam-beam effect is the major source of nonlinearities in high-energy colliders. Such nonlinearities impose certain limits on the collider luminosity due to beam instability. Even if the long-range beam-beam interaction could be avoided in some crossing schemes, the head-on beam-beam instability remains as the most fundamental luminosity limitation for proton-proton colliders. The strongly nonlinear beam-beam force excites high-order betatron resonances, so particles diffuse into the tails of the transverse distributions and get lost. For the Superconducting Super Collider (SSC) the beam-beam interaction luminosity limit is about $3 \times 10^{34} \mathrm{~cm}^{-2} \mathrm{~s}^{-1}$, i.e., well above the design luminosity of $10^{33} \mathrm{~cm}^{-2} \mathrm{~s}^{-1}$. However, the tune spread generated by head-on beam-beam interactions causes fast decoherence of the betatron oscillations [1] and, therefore, imposes more stringent requirements on any feedback system. This is especially important for such a low-emittance machine as the SSC. For the Large Hadron Collider (LHC), with its design luminosity of $1.6 \times 10^{34} \mathrm{~cm}^{-2} \mathrm{~s}^{-1}$ and a lower energy, a solution for compensating the head-on beam-beam effect is even more important.

\section{Scheme of compensation}

The head-on beam-beam effect in proton-proton machines is the defocusing of particles of one bunch by the space charge of the counterrotating bunch. It might be compensated, under certain conditions, by collisions of the bunch on each turn with a space charge of the opposite sign-for example, with a low-energy electron beam. This assumes that we are still far away from the conditions of one-pass collective instabilities [2].

A simple and reasonable approach to the calculation of the head-on beam-beam effect for the case of a relatively low beam intensity is the so-called weak-strong model. In this model one beam is regarded as "weak" and the counterrotating beam, unperturbed by the weak beam, is considered as 
"strong." If the particle distribution of the counterrotating ("strong") beam is a round Gaussian, the kicks given to the protons of the "weak" beam by the space charge of the "strong" beam are [3]:

$$
\left[\begin{array}{l}
\Delta \mathrm{X}^{\prime} \\
\Delta \mathrm{Y}^{\prime}
\end{array}\right]=\frac{2 N_{\mathrm{b}} r_{\mathrm{p}}}{\gamma_{\mathrm{p}}} \frac{1}{\mathrm{X}^{2}+\mathrm{Y}^{2}}\left(1-\exp \left(-\frac{\mathrm{X}^{2}+\mathrm{Y}^{2}}{2 \sigma^{2}}\right)\right)\left[\begin{array}{l}
\mathrm{X} \\
\mathrm{Y}
\end{array}\right],
$$

where $N_{\mathrm{b}}$ is the number of particles in a bunch of the strong beam, $r_{\mathrm{p}}$ the classical proton radius, $\gamma_{\mathrm{p}}$ the Lorentz relativistic factor of a $20-\mathrm{TeV}$ proton, and $\sigma$ the rms beam size at the low- $\beta$ IPs.

An ideal solution for compensation of the beam-beam effect in proton-proton machines is an instantaneous collision of a proton bunch with a counterrotating beam of negatively charged particles having the same parameters as a counterrotating proton bunch. In this case the angular kick delivered to a primary proton by the space charge of the counterrotating proton bunch would be exactly canceled by the kick delivered by the space charge of the compensating beam. A low-energy electron beam could be used as the compensating beam. It is important that the compensating beam be formed with the same two-dimensional transverse coordinate distribution as the proton bunch. The longitudinal profile of the compensating beam is not really important, because the angular kick delivered to the primary proton by the compensating beam could be accumulated along the length of the available collision region (about $2 \mathrm{~m}$ for the SSC case), which is still short in comparison with a betatron wavelength.

Instead of placing a compensating collision point immediately after the proton-proton collision, one can place the collision point in a more accessible location with a betatron phase advance relative to the proton-proton collision point of $n \pi$, where $n$ is an integer, the same in the $\mathrm{X}$-plane and in the Y-plane. Here the image of the proton beam in the X-Y plane is similar to the image in the proton-proton interaction point, differing only in scale. By using a place in the lattice with high beta values, one could relax the requirement to form a beam of a very small size, as in the 
low- $\beta$ IPs. In the SSC case, a beam with sigma of $0.2 \mathrm{~mm}$ could be used, for example, in the East Cluster, close to the interaction points. Because of a half-integer phase advance between two high-luminosity interaction points at the SSC, two separate compensating devices in each ring should be used to compensate full beam-beam interaction in the two low- $\beta$ IPs.

The current in the relativistic electron beam that is necessary for compensation of the beam-beam effect of the counterrotating beam should be about equal to the current of the proton beam, i.e., $75 \mathrm{~mA}$ in the case of the SSC baseline design. More precisely, for 10-keV electrons and a 2-m collision region, this current for the SSC would be about $32 \mathrm{~mA}$. Electron guns with comparable parameters are available now from industry. Deviation of the intensity of the individual proton bunches from the average value, if large, could be compensated by strobing the electron beam in time, using available bunch-by-bunch intensity information.

\section{Behavior of low-energy electrons inside the proton bunch}

One of the problems with using a low-energy electron beam for beam-beam effect compensation is electron oscillations during passage through the proton bunch. Even passing once and then being dumped, electrons experience some oscillations inside the proton bunch, which makes it difficult to distribute proper kicks among all the protons in the bunch. Figure 1 presents a trajectory of a $10-\mathrm{keV}$ electron with an impact parameter of $100 \mu \mathrm{m}$ colliding with a bunch of $10^{10}$ protons. The space distribution of protons is three-dimensional Gaussian with $\sigma_{\mathrm{x}}=\sigma_{\mathrm{y}}=$ $100 \mu \mathrm{m}, \sigma_{z}=50 \mathrm{~mm}$, which represents the typical parameters of the SSC beam. The ZBEAM tracing code used is described elsewhere [4]. To simplify the calculations, only transverse components of the electrical field of the bunch were taken into account. This is a good approximation for a long bunch with small transverse dimensions. Because of the low energy of the electrons we neglect possible radiation effects. 
As seen in Figure 1, a 10-keV electron makes several oscillations before it leaves the proton bunch. This immediately imposes difficulties in delivering the proper kick to all the protons in the bunch, because the distribution of electron density in the bunch will vary along the bunch length. (Some kind of pinch effect occurs.)

Three methods were considered to avoid this difficulty. First, one can use electron beams with different energies to vary the pinch frequency and distribute the negative charge inside the proton bunch more uniformly. Second, one can try to introduce some angular spread in the electron beam to smear the pinch effect. Third, a solenoidal magnetic field could be used to prevent electron oscillations. It was found that the solenoidal field option presents the best solution to the problem.

Figure 2 presents the trajectory of a $1-\mathrm{MeV}$ electron inside the proton bunch. One can see that the period of oscillation is different from that presented in Figure 1(b) for 10-keV electrons. Therefore, by mixing the effects of two beams one can hope to achieve a proper compensation of the beam-beam effect. Figure 3 gives a representation of what happens when an angular spread of $1^{\circ}$ is introduced in the vertical or horizontal planes. Some smearing of the pinch effect is obvious.

However, the best way to avoid the pinch effect is to use a solenoidal magnetic field directed along the beam in the compensating interaction region. Figure 4 presents the cases when such a field is applied. One can see from Figures $4(\mathrm{~b})$ and $4(\mathrm{c})$ that in the case of $\mathrm{B}=2 \mathrm{~T}$ the radial position of a $10-\mathrm{keV}$ electron with zero incoming angle remains constant with an accuracy of about $1 \mu \mathrm{m}$. Even after introducing an angular spread of $1^{\circ}$ the radial positions of the electron remain practically constant. Therefore, for a round Gaussian beam a collision of a proton bunch with a low-energy electron beam kept stable by a solenoidal magnetic field will adequately approximate a proton-proton collision with the opposite sign of the effect. 


\section{Configuration of the device}

Figure 5 presents a design for a possible device for beam-beam effect compensation. A low-energy electron beam, being kept transversally stable by the solenoidal magnetic field, is directed by the deflecting magnets to the interaction region to produce a head-on collision with the proton bunch. The influence of the solenoidal magnetic field on the proton bunch is then compensated by the same field configuration with the opposite polarity.

We believe that in this way it is possible to reduce the head-on beam-beam effect in practice by one or two orders of magnitude, and to improve considerably the high-luminosity performance of future proton-proton colliders.

\section{Acknowledgments}

The authors would like to thank G. Dugan, D. Johnson, D. Larson, and T. Toohig for valuable discussions.

\section{References}

[1] E. Tsyganov, H.-J. Shih, R. Meinke, W. Nexsen, M. Herath-Banda, and A. Taratin, "Decoherence of the SSC Beam Oscillation Due to Head-On Beam-Beam Collisions," SSCL-Preprint-481, July 1993.

[2] N. A. Solyak, "Collision Effects in Compensated Bunches of Linear Colliders," INP-88-44, March 1988, Novosibirsk. V. E. Balakin and N. A. Solyak, "VLEPP Beam-Beam Effects," Proceedings of the XIII International Conference on High Energy Accelerators, 1986, v. 1, p. 151, Novosibirsk.

[3] L. R. Evans, "The Beam-Beam Interaction," in CERN Accelerator School on Antiprotons for Colliding Beam Facilities, CERN Report 84-15, p. 319, 1984.

[4] E. Tsyganov and A. Zinchenko, "Charge Tracing Code for the SSC Environment," SSCL-618, March 1993. 
(a)

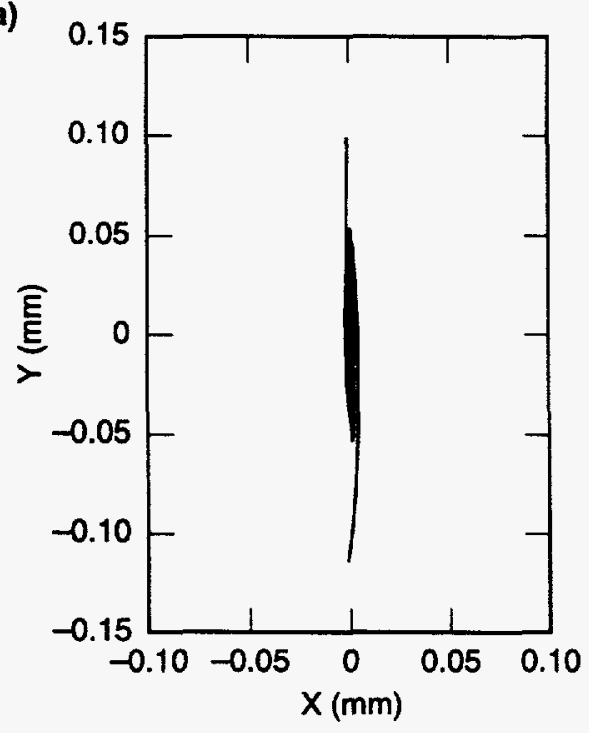

(b)

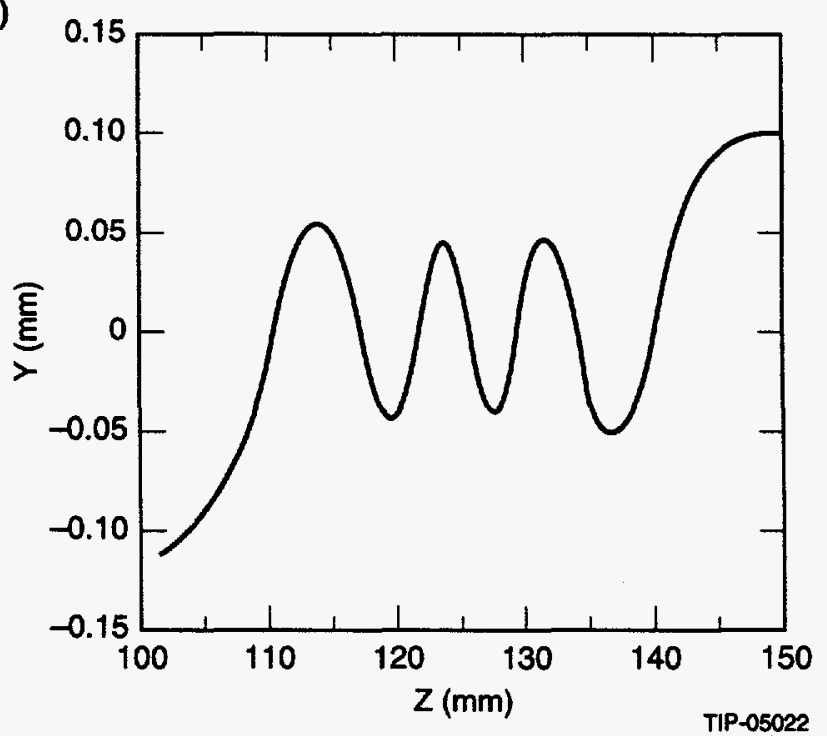

Figure 1. The trajectory of a $10-\mathrm{keV}$ electron with an impact parameter of $100 \mu \mathrm{m}$ colliding with a bunch of $10^{10}$ protons, according to the tracing code ZBEAM. The proton bunch is Gaussian in three dimensions with $\sigma_{x}=\sigma_{y}=100 \mu \mathrm{m}, \sigma_{z}=50 \mathrm{~mm}$. (a) X-Y view; (b) $\mathrm{Y}-\mathrm{Z}$ view. The proton bunch (not shown) is moving to the right, and the electron is moving to the left. Plus/minus $3 \sigma$ of the proton bunch charge distribution in the Z-direction is treated by the tracing code. The time interval used in the calculations is 1 ps. 


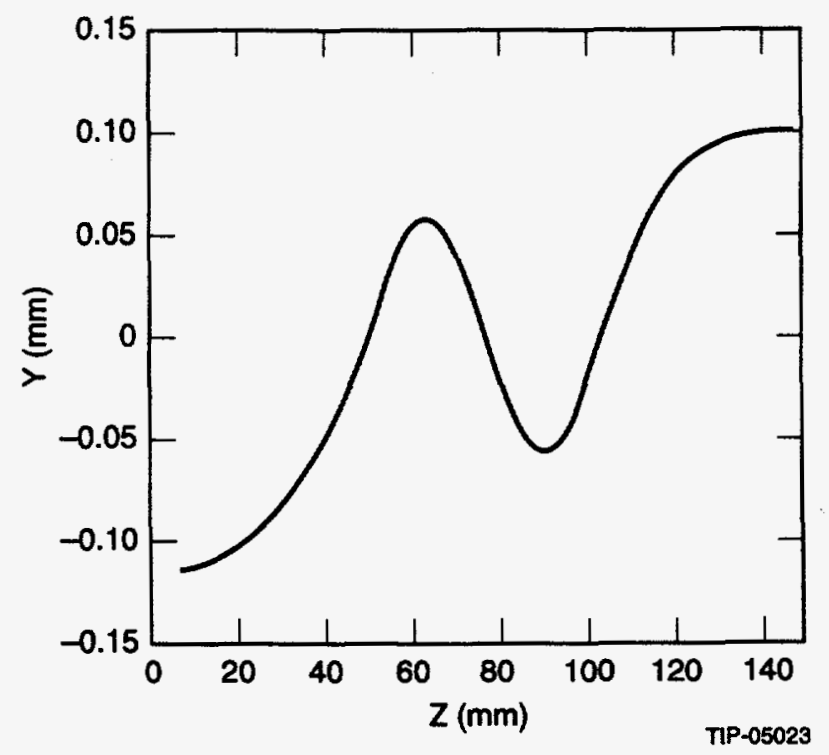

Figure 2. The trajectory of a 1-MeV electron during its interaction with a proton bunch, $\mathrm{Y}-\mathrm{Z}$ view. Other parameters as in Figure 1.

(a)

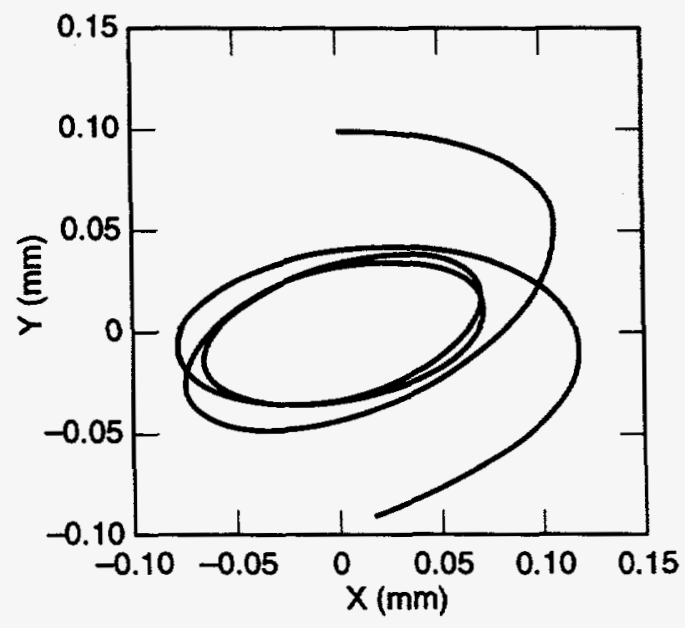

(b)

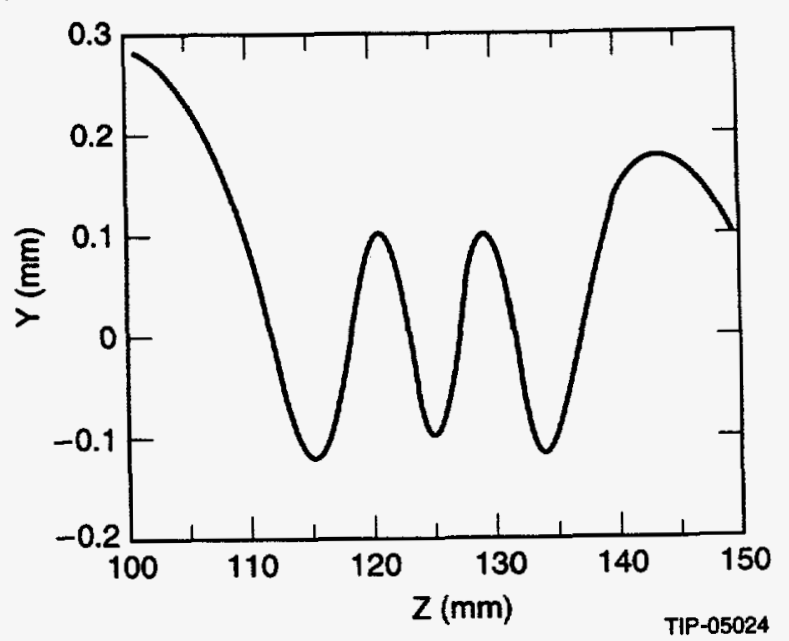

Figure 3. Trajectories of $10-\mathrm{keV}$ electrons with non-zero incoming angles inside the proton bunch. (a) $\phi=0^{\circ}, \theta=+1^{\circ}, X-Y$ view; (b) $\phi=90^{\circ}, \theta=+1^{\circ}$, Y-Z view. 
(a)

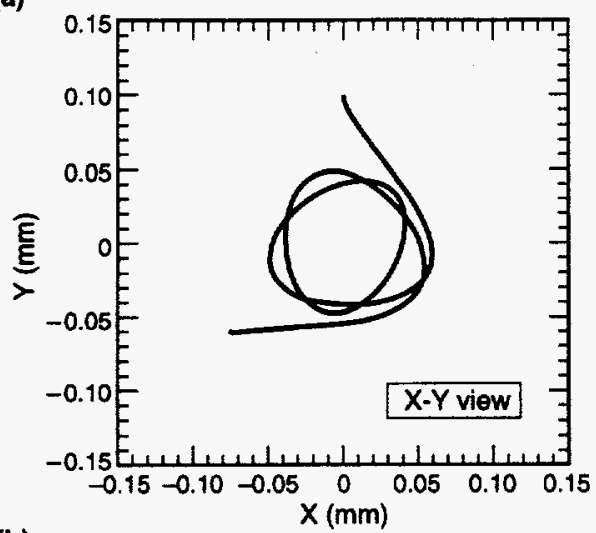

(b)

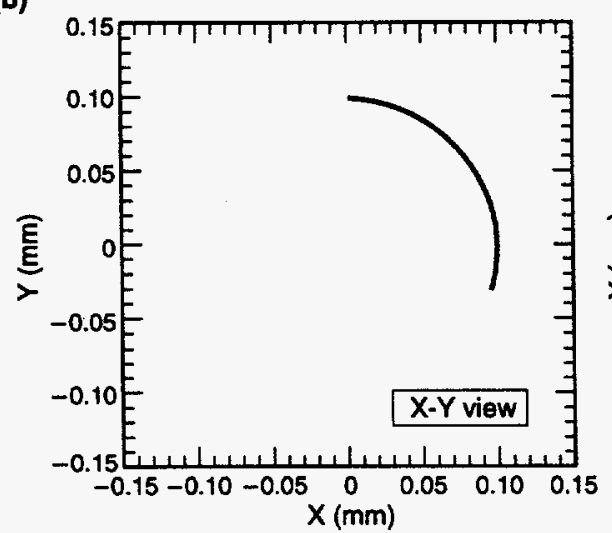

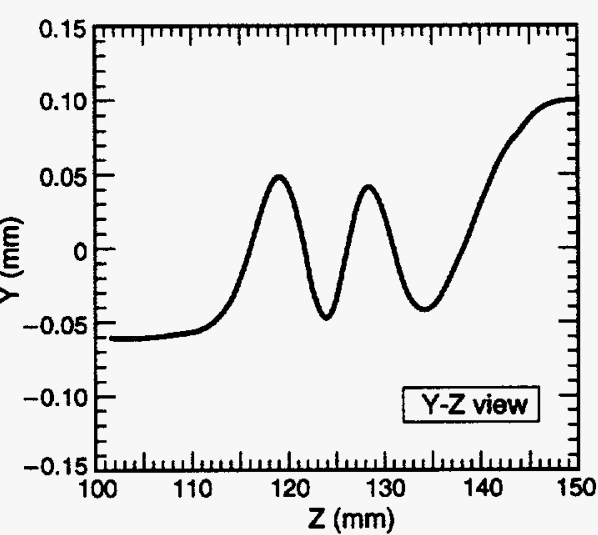

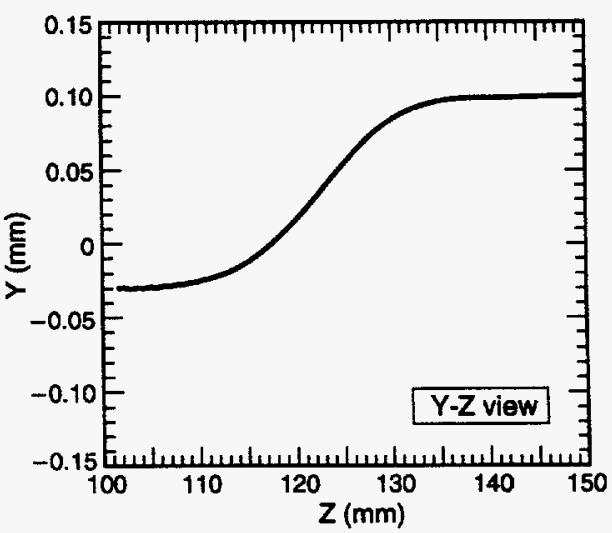

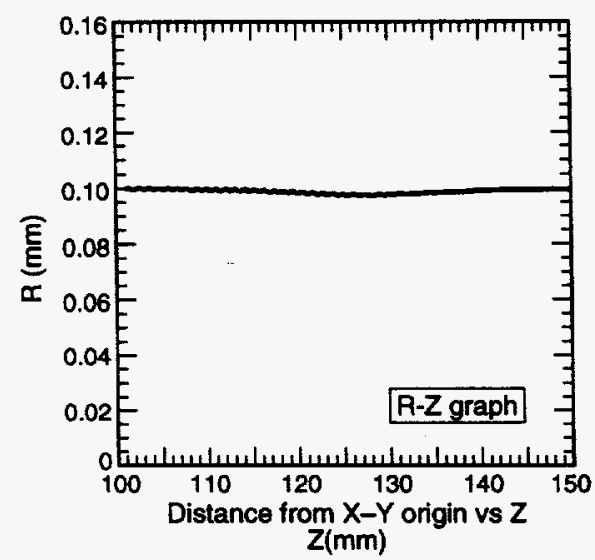

Figure 4. The trajectory of a $10-\mathrm{keV}$ electron with an impact parameter of $100 \mu \mathrm{m}$ in the proton bunch when a solenoidal magnetic field is applied: (a) solenoidal magnetic field of $0.1 \mathrm{~T}$, projections $\mathrm{X}-\mathrm{Y}$ and $\mathrm{Y}-\mathrm{Z}$; (b) solenoidal magnetic field of $2 \mathrm{~T}$, projections $X-Y$ and $Y-Z$. The graph of $R-Z$ is also presented, $R=\sqrt{X^{2}+Y^{2}}$; (c) histogram of $R$-measurements for the case of $2 T$. The mean value of $R$ is about $99 \mu \mathrm{m}$, the rms value is about $0.7 \mu \mathrm{m}$; (d) $\phi=0^{\circ}, \theta=+1^{\circ}$, solenoidal magnetic field of $2 \mathrm{~T}, \mathrm{X}-\mathrm{Y}$ view; (e) $\phi=90^{\circ}, \theta=+1^{\circ}$, solenoidal magnetic field of $2 \mathrm{~T}, \mathrm{X}-\mathrm{Y}$ view; (f) histogram of $R$-measurements for case (e). The mean value of $R$ is about $99 \mu \mathrm{m}$; the rms value is about $2 \mu \mathrm{m}$. 
(c)

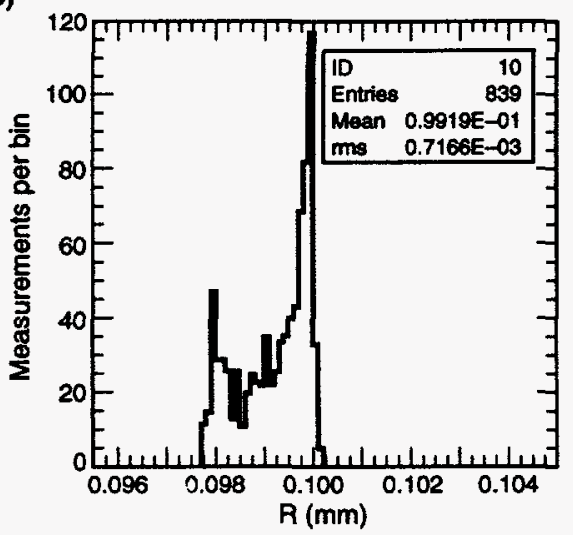

(e)

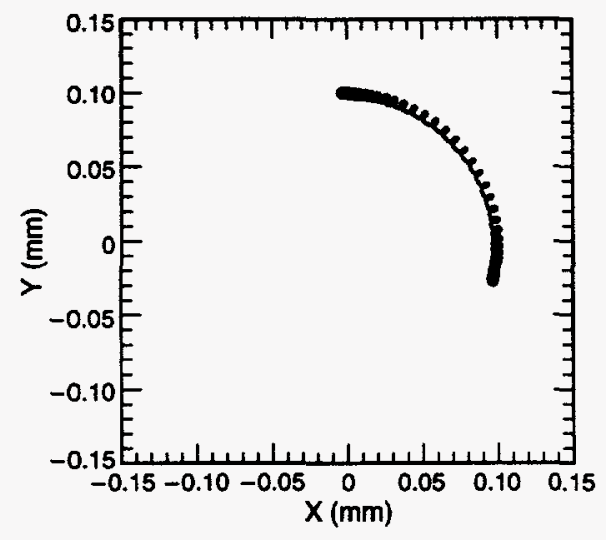

(d)

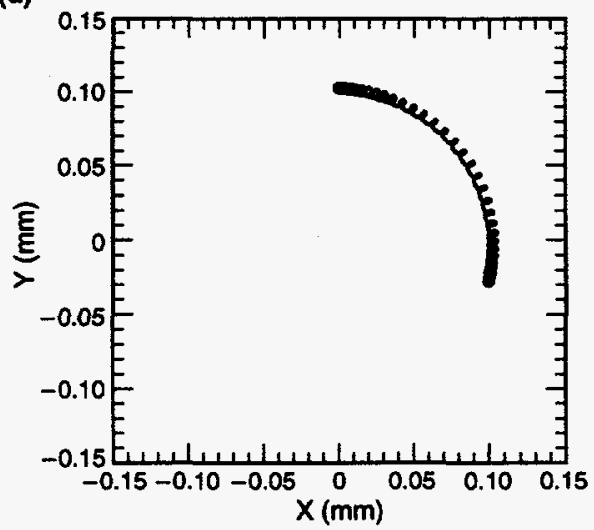

(1)

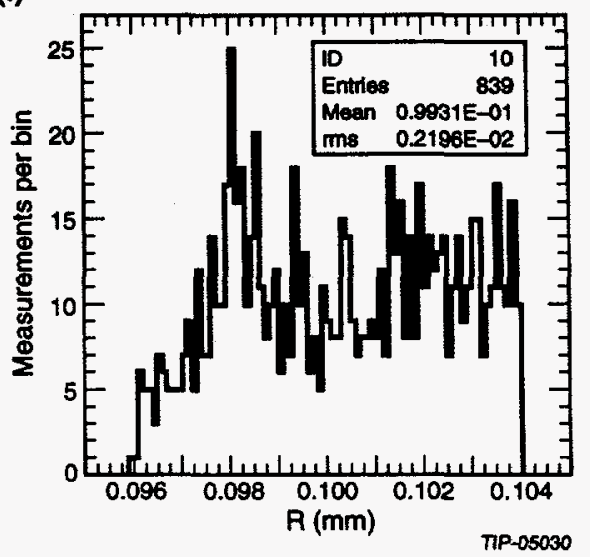

Figure 4. (Cont.) 


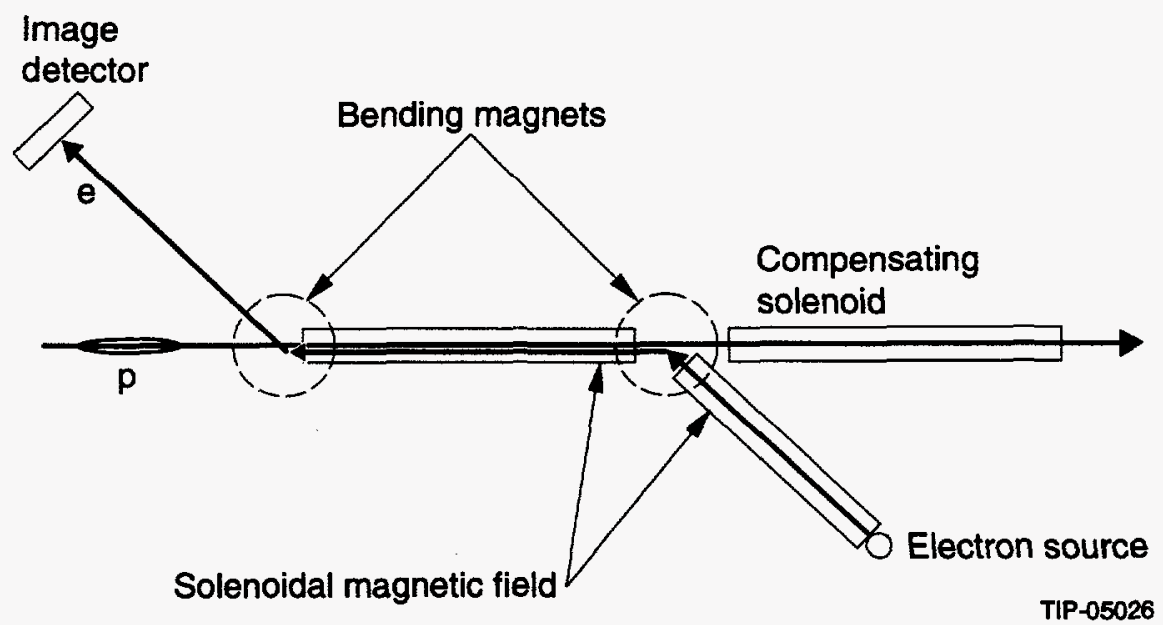

Figure 5. Schematics of a beam-beam compensating device. A low-energy electron source collides with a bunch of protons. Electrons are kept stable in space by a solenoidal magnetic field. After collision with the proton bunch, an electron beam is deflected to the image detector, which is used to steer the electron beam relative to the proton bunch. 